\section{Optical Dispersion for Coals}

Usnve the apparatus previously described ${ }^{1}$, together with a set of Wratten Monochromat light filters Nos. 70-75, it has been possible to examine the variations of refractive index over a limited range of wave-lengths in the visible spectrum for several typical coals of varying rank. The Brewster angle was determined with each filter placed in turn before the light source, and the corresponding refractive index calculated. The determining factor limiting the accuracy of the measurements was the stability of the photo-cell amplifier. Under the most unfavourable conditions the limits of accuracy of the determination of refractive index were $\pm 0 \cdot 01$. In general, the accuracy was greater than this.

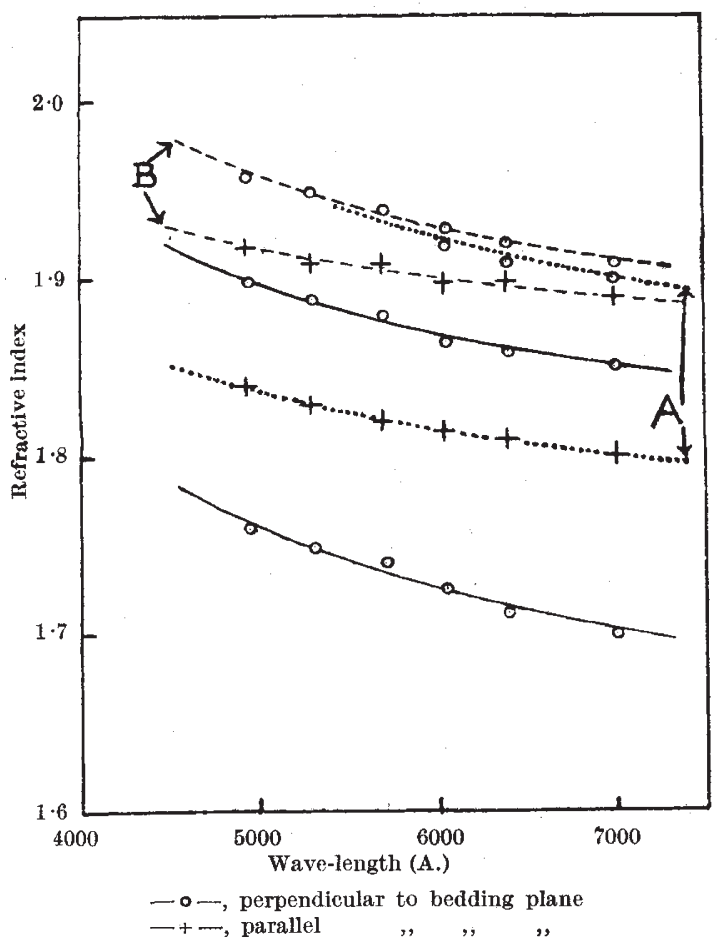

Typical results are given in the accompanying figure. For two isotropic coals, the lower full-lithe curve is for a free-burning coal and the upper for a coking coal. For the anisotropic coals measurements were made in directions perpendicular (rings) and parallel (crosses) to the bedding plane. The curves $A$ are for a Welsh anthracite and the curves $B$ for a Welsh steam coal.

For all the measurements made in a direction perpendicular to the bedding plane the dispersion curves are approximately parallel; the curves for the measurements made parallel to the bedding plane are themselves parallel. But the slopes of the two sets of curves, shown respectively with rings and crosses; are markedly different. From these results it would appear that the refracting. material varies but little with the rank of the coal. The difference shown in the two directions would, however, be consistent with a large-scale structure in which anisotropic units occur with preferred orientation. On such a supposition the isotropy of the lower rank coals could be obtained by completely random orientation of the same anisotropic units.

Thanks are due to Mr. J. G. Bennett, director, and Dr. D. H. Bangham, principal scientific officer, of the British Coal Utilization Research Association, for their kindness in providing the specimens.
Physics Department,
Chelsea Polytechnic,
C. G. Cannon.

London, S.W.3.

${ }^{3}$ NATURE, 150, 690 (1942); and 151, 53 (1943).

\section{Nature of Entropy}

THE opening paragraph of Mr. Ian Campbell's letter ${ }^{1}$ on the nature of entropy reads somewhat strangely to one fresh from the perusal of "Physics and Philosophy" by Sir James Jeans. Campbell argues that "a quantity which fits so completely into the mathematics of the subject must surely have some relation to the physical facts", and suggests that entropy may be regarded as analogous to momentum in mechanics. Jeans asks whether we should adopt mechanical explanations of Nature or the mathematical description of Nature, and concludes: "whether the ghostly remains of matter should be labelled as matter or as something else is mainly a question of terminology" (p. 216).

From the educational point of view, however, it is in my opinion necessary that the beginner in a scientific subject should be given at the outset some familiar mental picture. Some physical analogies of the "ghostly" quantity entropy have been described in a recent text-book ${ }^{2}$, and it may be pointed out that before the end of last century it was customary in the Royal Naval Engineering College, Devonport, to regard entropy as 'thermal inertia', and to use rotational motion especially to illustrate thermodynamic principles, treating entropy as 'rotational inertia' (moment of inertia) ${ }^{3}$. In his presidential address to the Physical Society in 1911, Prof. H. L. Callendar stressed the correspondence between entropy and 'caloric'. On this view, we may regard Joseph Black's 'matter of heat' as equivalent to a mathematical function, or alternatively we are in some measure entitled to regard entropy as the modern expression for caloric.

In order to support his view as to the nature of entropy, Campbell proposes the adoption of a new scale of temperature so that "temperature may become proportional to the square root of heat energy and, in the kinetic theory, to the mean molecular velocity". I am not at all clear as to his attitude to Lord Kelvin's absolute scale of temperature-one sentence in his letter asserts, or at least suggests, that temperature "cannot be measured absolutely". On this subject there is a recent articles on a dynamical treatment of the elements of heat by Dr. G. Burniston Brown, in which an elementary and more directly empirical discussion is given in a form suitable for beginners. In this connexion it is worth while directing attention to the fact that momentum, depending on the first power of the velocity, is a vector quantity, having direction and sense as well as magnitude. This in itself indicates that momentum is not likely to be a suitable analogy for entropy. It should be noted that in the elementary development of Boyle's law on the basis of the kinetic theory of gases, the square of the velocity is involved because not only momentum enters but also 